\title{
The Effect of Spatial Tasks on Visually Impaired Peoples' Wayfinding Abilities
}

\author{
Mark Blades, Yvonne Lippa, Reginald G. Golledge, R. Daniel \\ Jacobson, and Robert M. Kitchin
}

Abstract: Thirty-eight people with visual impairments learned a 483-meter novel route through a university campus in four groups: verbalization, modeling, pointing, and control. The performance of all four groups improved with greater experience of the route, but the modeling group improved more than did the control group.

W ayfinding-the ability to learn a route and retrace it from memory-is one aspect of people's cognitive map knowledge, which includes all aspects of encoding, processing, and retrieving information about the environment (see Golledge, 1999; Kitchin \& Blades, 2001; Kitchin \& Freundschuh, 2000). This article reports on a study that investigated how people with visual impairments (those who are blind or have low vision) learned a route through an unfamiliar environment.

Although there have been a few studies of route learning by people with visual impairments, many of these studies have been conducted in small and limited environments (see Ungar, 2000). These studies have been important for highlighting specific aspects of cognitive map behavior, but they have provided little information about how visually impaired people learn routes in large and complex environments. It is

The authors thank Michael Kinsella, Sarah Battersby, and Jim Marston for conducting the experiment and helping to code the data. only comparatively recently that researchers have begun to investigate the performance of visually impaired people in such environments. These studies are summarized next.

\section{Review of research}

Ochaíta and Huertas (1993) tested the ability of 17 year olds with visual impairments to learn a route between seven landmarks placed in a public square. The participants were given one guided experience along the route and then three or four trials in which they walked the route on their own. At the end of the trials, they were asked to make a scale model of the layout of the landmarks and give verbal estimates of the distances between them. The participants were accurate at making the model and estimating the distances. This finding suggests that visually impaired adolescents are capable of learning a route and its configuration after several repeated trials. Espinosa, Ungar, Ochaíta, Blades, and Spencer (1998) conducted two studies of route learning in real environments. In one study, visually impaired adults walked a 
$1.2 \mathrm{~km}(0.75$ miles) route through an unfamiliar suburban area. After one guided experience, they had two trials in which they walked the route on their own, and the number of times they deviated from the route was noted. By the second trial, the participants' performance was very good, with less than one error per route. In the second study, Espinosa et al. (1998) asked visually impaired adults to walk a $2 \mathrm{~km}$ (1.25 miles) route through the Madrid city center. The participants had one guided experience of the route and then retraced it on their own in three subsequent trials. By the last trial, they made only two deviations from the route. The studies by Ochaita and Huertas (1993) and Espinosa et al. (1998) showed that visually impaired participants can learn routes through real environments effectively. However, neither study reported the participants' performance on individual trials or included a control group of sighted participants. Therefore, it is not possible to know how quickly the participants learned the routes or how this learning would compare with that of sighted participants.

One study included a sighted control group. Passini and Proulx (1988) compared how totally blind and sighted people learned a 250 -meter ( 273 yards) route along corridors on two floors of a university building. The participants were given two guided experiences along the route and then walked it on their own. Overall, the totally blind group made four times as many errors and hesitations as did the sighted group when walking the route on their own. Nonetheless, a third of them retraced the route without error, and a third were able to make a completely accurate map of the route. This finding indicates that some people who are totally blind can learn routes in large environments as effectively as can sighted people. However, the participants in Passini and Proulx's study retraced the route only once on their own, so they had no opportunity for further learning.

These studies all suggest that route learning by visually impaired people in large environments can be effective, but as was pointed out, there are limitations to all the studies. To overcome these limitations, the authors conducted two studies of route learning in which the performance of visually impaired groups was compared to a control group of sighted participants over several learning trials.

The first study (Jacobson, Kitchin, Garling, Golledge, \& Blades, 1998) was carried out in Belfast, Ireland, where groups of participants who were totally blind or sighted or had low vision learned a novel $1.6 \mathrm{~km}$ (1 mile) route that included up to 16 choice points (e.g., left turns, right turns, and road crossings). The participants were guided along the route once by the experimenter. During the course of this guided experience, they were not given any information about the route or advice about how to learn it. They were just told that they would be expected to retrace the route another three times on their own. These were the three trials. During each trial, the participants' accuracy at each choice point was noted, and the participants' knowledge of the route was tested by asking them to point between places on the route, give a verbal description of it, and make a "model" of the route using magnetic pieces on a metal board. The study found that the participants who were blind or had low vision took slightly longer than the sighted participants to learn the routes, but by the third trial, all the groups could retrace the route 
almost without error. Furthermore, by the third trial, there was no difference in accuracy between the performance of the sighted and the two visually impaired groups on any of the three spatial tests (verbalizing, pointing, or modeling).

In the second study (Golledge, Jacobson, Kitchin, \& Blades, 2000), the authors repeated the procedure of the Belfast experiment in Santa Barbara, California, and the pattern of results in Santa Barbara was the same as was found in Belfast. They therefore concluded that in real-world contexts, people who are visually impaired can learn novel environments quickly and effectively and that they need only one or two additional experiences (more than sighted people) to acquire sufficient route information for independent travel. The findings of Jacobson et al. (1998) and Golledge et al. (2000) support the results of Ochaíta and Huertas (1993) and Espinosa et al. (1998) that people who are visually impaired can learn routes successfully.

The totally blind participants in Passini and Proulx's (1988) study did not perform as well (compared to the sighted participants) as did the visually impaired participants in the authors' studies, perhaps because the participants in Passini and Proulx's study were given only one trial on their own. As the authors found, participants who are visually impaired may require one or two additional trials to reach the same level as do sighted people. Nonetheless, the authors emphasize that only a small amount of additional experience is needed and that visually impaired people have the potential to learn new environments quickly.

The claim that people who are visually impaired can learn routes almost as quickly as can sighted people is based on the two studies just described (Golledge et al., 2000; Jacobson et al., 1998). However, during the debriefing sessions and followup interviews after those studies, the authors noted that some of the visually impaired participants said they were surprised at how well they had learned the route. They thought that the intensive testing during the experiment (i.e., the pointing, modeling, and descriptions they did on every trial) had contributed to their success. If this intuition was correct, it has two important implications.

The first implication is a methodological one. The fact that the performance of the participants with visual impairments was enhanced by greater practice could be due not only to repeated exposure to the route, but to the repeated assessments of their spatial abilities. Other researchers have pointed out that there are marked individual differences in the route learning and mobility of people with visual impairments (ThinusBlanc \& Gaunet, 1997; Ungar, 2000). These researchers have suggested that some differences may be due to the different strategies that individuals adopt in spatial learning tasks. For example, Ungar, Blades, and Spencer (1997) showed that visually impaired participants used a variety of different spatial strategies in a tactile maplearning task and that the type of strategy had an effect on the participants' success. Thus, it is possible that the repeated completion of spatial tasks in the authors' routelearning studies may have resulted in all the participants being given, in effect, optimal strategies for performing the route-learning task.

This methodological issue goes beyond the studies just described because in many cognitive map and environmental learning 
studies, the measures that are used may confound changes in participants' cognitive maps (for a further discussion, see Kitchin \& Blades, 2001; Kitchin \& Jacobson, 1997). The authors thought it was important to investigate the effects of test measures on route-learning performance, and this was one of the reasons for the experiment described in this article.

The second implication of the authors' previous studies is related to mobility instruction. Mobility specialists often use techniques like giving a verbal description of a route or pointing between places during mobility instruction, and they may also sometimes include modeling in such instruction. However, no previous investigation has compared these techniques to find out whether any are more effective than others for learning a new route.

\section{Aims and hypotheses}

In sum, the aim of the experiment was to find out the extent to which spatial tasks, such as pointing, describing, and modeling a route, facilitate route learning and whether one of these spatial tasks is more effective than the others. Participants who were blind or had low vision learned an unfamiliar route through a university campus by walking the route several times.

Each participant had a guided experience of the route and then walked it three times on his or her own. There were four groups of participants. One group carried out a pointing task while walking the route, one group gave a verbal description of the route after completing it, and one group did a modeling task each time. A fourth (control) group learned the route without carrying out any tasks.
The authors predicted that there would be no difference between the four groups' recall of the route the first time that they walked it on their own because the participants completed the spatial tasks only during the first trial (in the pointing condition) or at the end of that trial (in the verbalization and modeling conditions). However, if the tasks did contribute to learning, the authors expected that the three groups who carried out the spatial tasks would recall the route better than the control group the second and third times they walked along it. In other words, if the tasks contributed to learning, the authors expected an interaction between trial and group. No predictions were made about which of the spatial tasks would yield the largest contribution to learning. Given the results of their previous studies (e.g., Golledge et al., 2000; Jacobson et al., 1998), the authors did not expect differences in the performance of the two groups of visually impaired participants.

\section{Method}

\section{PARTICIPANTS}

Prior to the experiment, the participants with visual impairments were given a questionnaire about their vision and were asked whether they could see trees, sidewalks, or people in any light conditions. The participants who replied no for all these items were classified as severely visually impaired, and those who could see one or more of these items were classified as moderately visually impaired. The authors believe that this functional definition was an appropriate way to categorize the participants because route learning is likely to be most affected by whether participants can or cannot see landmark information. 
The participants were also asked to rate their confidence in traveling, on a scale of from 1 to 5 (very unsure, unsure, undecided, confident, and very confident) in different contexts (at home, on local streets, on busy roads, when crossing at traffic lights, at crossings without signals, in new environments, when making detours around unknown hazards, and when exploring away from a known route). If the participants' mean confidence rating was 2.5 or higher, they were classified as having "high" travel confidence, and if it was less than 2.5 , they were classified as having "low" confidence. The participants were also asked how much mobility instruction they had received by indicating one of four options: none, little, partial, or extensive.

Thirty-eight participants completed the experiment. The mean age of the participants was 54 years (range 18 to 82 years). The mean number of years of visual impairment was 26 (range 1 to 73 years). Ten participants were congenitally visually impaired, and 28 were adventitiously visually impaired. Three other participants also took part, but one could not complete the route because of construction work on the route and two found the task too difficult. The 38 participants were assigned to one of the four conditions; there were 10 each in the modeling and verbalization groups and 9 each in the pointing and control groups. There were no significant age differences among the four conditions, and no significant difference in the years of visual impairment among the conditions.

The participants were assigned to the conditions so that in each condition approximately (1) half the participants were men and half were women; (2) half the partici- pants were severely visually impaired and half were moderately visually impaired; (3) there were the same proportion of participants with high and low travel confidence; and (4) there were the same proportion with no, little, partial, and extensive mobility instruction. As far as possible, given these constraints, the groups included both congenitally and adventitiously visually impaired participants. All but three of the participants used long canes, and six also used dog guides, but there were no more than two dog users in any one condition. In other words, the participants in each condition were as equivalent as possible.

\section{Procedure}

A 483-meter (528 yards) route was designed through the campus of the University of California, Santa Barbara (see Figure 1). The route followed a path between several buildings and groups of trees and bushes. There were three landmarks along the route: "café corner," "ivy corner," and "steps."

The experiment was carried out when there was little or no traffic on the route. Each participant met the experimenter and received the experimental instructions at an office away from the route. Then they were blindfolded and guided to the start of the route, where the blindfolds were removed. The participant was then guided along the route (hereafter referred to as the learning trial). The experimenter pointed out distinct characteristics of the starting point, oriented the participant in the direction of the route, and explained which landmark would be encountered first. The participant was then guided along the route solely by verbal instructions. The experimenter walked closely behind the participant and 


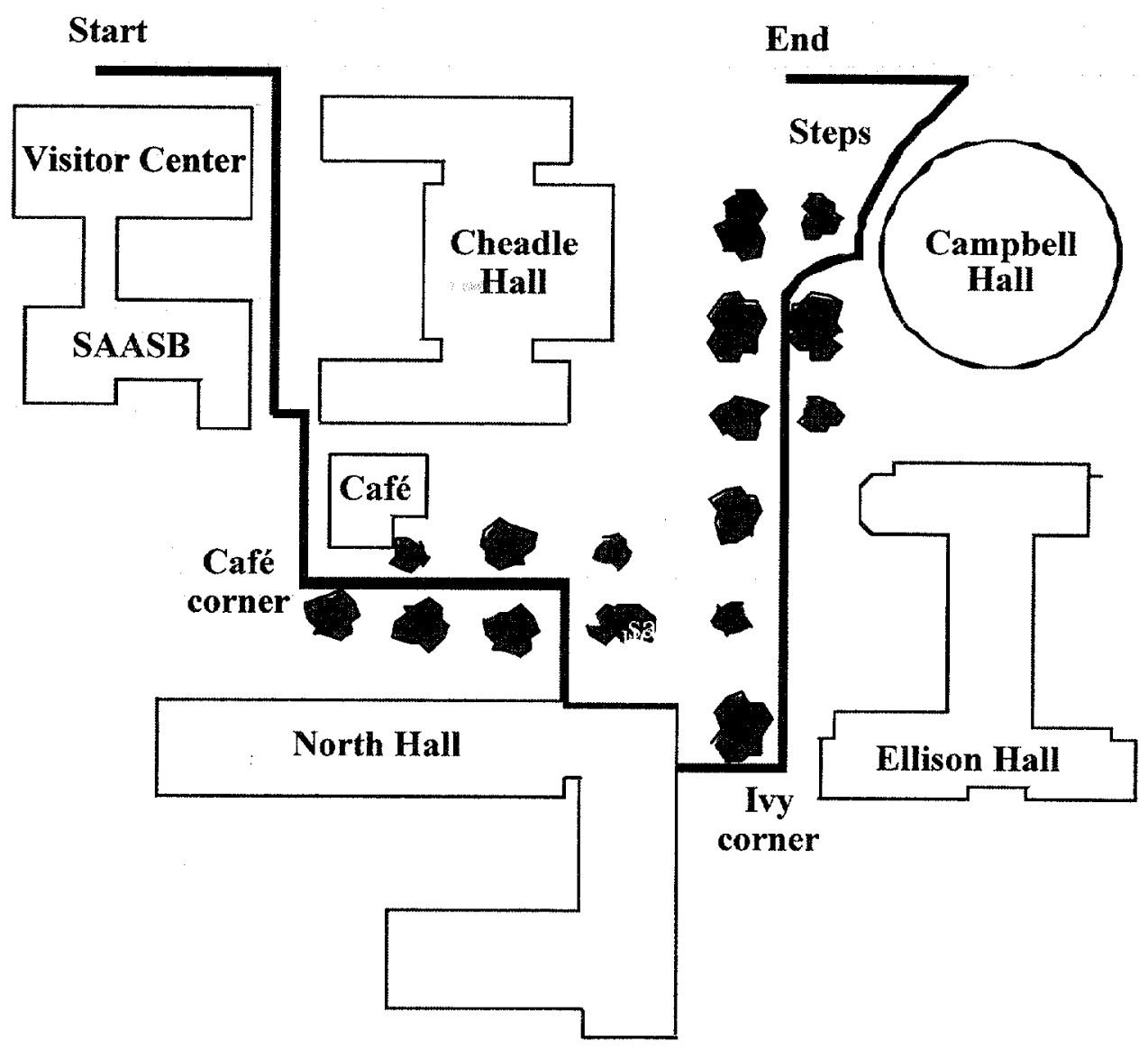

Figure 1. Plan of the route.

pointed out the characteristics of the route that could be picked up by shorelining or sweeping with a cane (e.g., the edge of a sidewalk, curbs, cracks, grass edges, the bottom of buildings, and signs). The participant was never given any directional cues or information. When the participant made turns, he or she were asked to make contact with an object at the turn (e.g., a building) and then go around the object. The participant was stopped at the first landmark (café corner) while the experimenter named it and indicated what the next landmark would be. The participant was then guided along the next segment of the route to ivy corner, where the naming procedure was repeated. This procedure was repeated for the last two segments of the route. Having walked the route, the participant was offered a short break. Then he or she was blindfolded and guided back to the start of the route (by a circuitous path) to begin the first trial.

In each trial, the participants were asked to retrace the route by themselves and to stop at and name each landmark as they 
reached it. They were assured that the experimenter would follow them to maintain their safety. The experimenter noted the walking time and any errors made by the participants, and an assistant videotaped each participant. If the participants went more than 6 meters ( 6.5 yards) in an incorrect direction, the experimenter stopped them. They were then told that they had gone in the wrong direction and were guided back to the point where they had made the error.

Each participant carried out three trials, with a short break between the trials. The participants were always blindfolded while they were led back to the start of the route by an indirect path. These procedures were common to all the trials, but further procedures depended on the condition, as described next.

\section{Conditions}

Control condition. The participants in this condition simply retraced the route. After reaching and naming a landmark, they immediately continued along the route. Apart from retracing the route on the three trials, they did not undertake any additional activities.

Verbalization condition. In this condition, the participants followed the procedure of the control condition, but at the end of each trial, they were asked to give a verbal description of the route with as much detail as possible. If a participant was unable to recall part of the route, the experimenter provided indirect cues (e.g., "Do you remember a sign?") but did not give specific directions.

Modeling condition. The procedure in this condition was also similar to the control condition, but at the end of each trial, the participants were asked to make a map of the route. They were given a metal board $(43.2 \mathrm{~cm} \times 28 \mathrm{~cm}$ or 17 inches $\times 11$ inches) and a set of magnetic pieces (short and long bars, short and long curves, circles to represent the start and end of the route, and squares to represent the landmarks) to make the model. There were more magnetic pieces than needed to model the route. Each finished model was photographed.

Pointing condition. The participants in this condition were asked to point between landmarks. For example, at the start of each trial, they were asked to point from the start to the three landmarks (café corner, ivy corner, and steps) and to the end of the route. At the café corner, the participants pointed to the other two landmarks and to the start and end of the route. This procedure was repeated at each pointing location, so that a total of 20 pointing estimates were made during each trial. The order of landmarks to which the participants pointed was random and was different for each location and each participant, but the random order remained the same over each participant's three trials. A compass was attached to a participant's pointing arm so that the experimenter could note the bearing accurately.

\section{Measurement of Performance}

The time to perform each of the spatial tasks was noted. Performance in the three spatial tasks was videotaped. The participants were not given any feedback on their performance.

Route-learning performance was measured in two ways: as the number of errors made while walking the route and the time taken to complete the route. Walking errors were coded as follows. While walking the route the experimenter made notes about the 
location and the type of the errors made by the participants. To code the errors, the experimenters identified 28 choice points along the route where the participants had to decide whether to go straight, to turn, or to stop. At each choice point, the participants' actions were classified in one of four categories: successful navigation (scored 1); a hesitation or deviation from the route that was self-corrected and followed by successful navigation (scored 2); a deviation from the route that needed guidance or a reminder for successful navigation (scored 3 ); or being lost, when the participants said they were lost or failed to find their way after repeated guidance (scored 4). In other words, a higher score represented more errors and poorer performance. Ten participants were chosen randomly and were scored by two raters in addition to the experimenter. For this sample, the agreement between the main rater and each of the other two raters was determined by Cohen's kappa. The two values were high, with kappa $=.87, p<.001$, and kappa $=.84, p$ $<.001$.

\section{Results}

The purpose of this experiment was to compare the participants' performance in the experimental conditions (in which the verbalization, modeling, or pointing tasks was completed) with the participants in the control condition (in which no spatial task was carried out). Performance was assessed by the number of walking errors and by the time taken to complete the route.

\section{WALKING ERROR}

A 4-condition (control, verbalization, and modeling versus pointing) $\times 3$-trial
(Trials 1 and 2 versus Trial 3) $\times 2$-vision (moderately versus severely visually impaired) analysis of variance (ANOVA) was carried out. There was an effect for trial $F(2,60)=34.20, M S E=0.03, p<$ .001 , and post hoc comparisons (all $p<$ $.001)$ showed that the performance on Trial 3 (mean score across choice points: 1.25), was better than on Trial 2 (mean score: 1.34), and the performance on Trial 2 was better than on Trial 1 (mean score: 1.50). In other words, there was a consistent improvement in route learning from the first trial to the third trial, and by the third trial, the participants' route knowledge was very good because their mean score was close to 1 (reflecting accurate performance at most choice points).

There was also an effect for vision, $F(1$, $30)=15.46, M S E=0.20, p<.001$, because the participants who were moderately visually impaired (mean score 1.20 ) performed better than those who were severely visually impaired (mean score 1.53). There were no interactions involving vision. In other words, the participants who were severely visually impaired performed more poorly than did those who were moderately visually impaired irrespective of trial or condition.

There was no effect for condition, $F(3$, $30)=1.23, M S E=0.20, p>.05$. The mean scores across choice points were 1.47 , $1.41,1.29$, and 1.27 for the control, verbalization, modeling, and pointing group, respectively. The interaction between trial and condition was not significant, but was close to the conventional level of significance, $F(6,60)=2.03, M S E=0.03, p=$ .095 (see Figure 2).

An examination of the pattern of performance at each of the 28 choice points along 


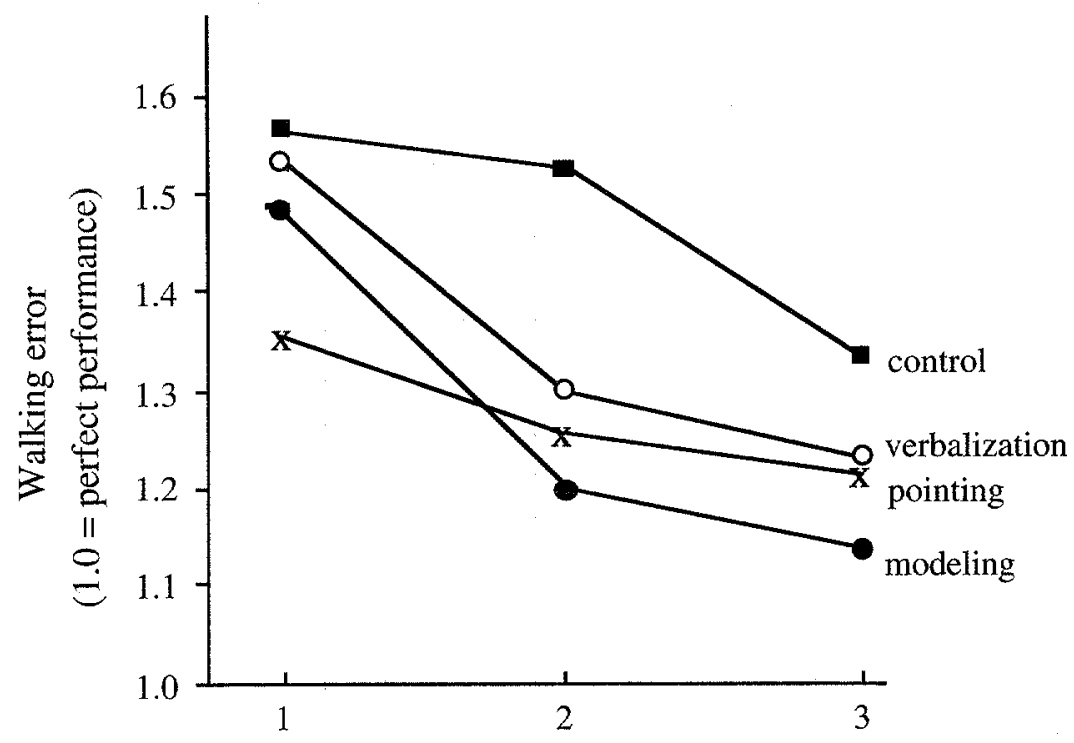

Test trial

Figure 2. Walking error for all choice points: Interaction between trial and condition.

the route showed that behavior at some choice points was less error prone than at others. The participants were quite successful at some choice points, even on the first trial. If they were successful at some choice points from the start of the experiment, there was little opportunity for them to improve on later trials, and this situation might have masked any learning differences. For this reason, the authors focused on the seven choice points where performance was particularly poor on the first trial, hereafter referred to as the seven critical choice points.

Performance at the critical choice points was analyzed using a 4-condition (control, verbalization, and modeling versus pointing) $\times 3$-trial (Trials 1 and 2 versus Trial 3) $\times 2$ vision (moderately versus severely visually impaired) ANOVA. This analysis showed that the pattern of performance at the critical choice points was the same as the pattern found in the analysis of all the choice points together. The participants' performance improved across the three trials, $F(2,60)=44.80, \mathrm{MSE}=0.09$, $p<.001$. The mean scores for Trials 1,2 , and 3 across critical choice points were $2.07,1.61$, and 1.49 , respectively. All were significantly different from each other $(p<$ $.001)$. The moderately visually impaired (mean score: 1.49$)$ performed better, $F(1$, $30)=12.14, M S E=0.50, p<.01$, than the severely visually impaired (mean: 1.96). Although the interaction between trial and condition was not significant, it approached conventional levels of significance, $F(6,60)=2.03, M S E=0.09, p=$ .086 (see Figure 3).

\section{WALKING TIME}

The time taken (in minutes and seconds) to walk the segments of the route (i.e., from the start to café corner, from café corner to 


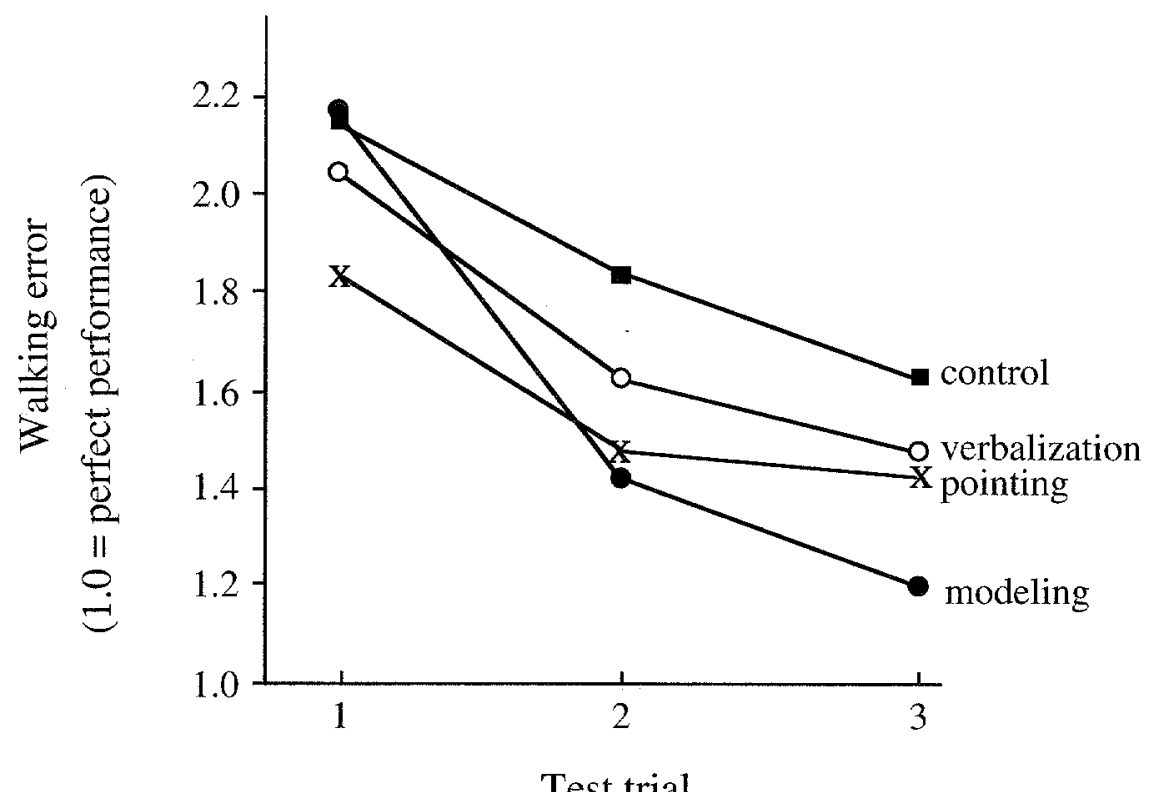

Figure 3. Walking error for critical choice points: Interaction between trial and condition.

ivy corner, from ivy corner to the steps, and from the steps to the end) was calculated. This procedure excluded the time spent at each of the landmarks because the time spent at these places varied, depending on condition. A 4-condition (control, verbalization, and modeling versus pointing) $\times 3$ trial (Trials 1 and 2 versus Trial 3 ) $\times$ 2 -vision (moderately versus severely visually impaired) ANOVA was carried out. There was an effect for trial, $F(2,60)=$ $31.10, M S E=7,860.55, p<.001$, because the participants became quicker over the three trials (12 minutes, 13 seconds, for Trial 1; 10 minutes, 52 seconds for Trial 2; and 10 minutes for Trial 3 ). The mean times were all significantly different from each other $(p<.001)$. There was also an effect for vision, $F(1,30)=13.96, M S E=$ $213,801.70, p<.01$, because the moderately visually impaired participants were faster ( 8 minutes, 19 seconds) and than were the severely visually impaired participants (13 minutes, 45 seconds). There were no other significant effects or interactions.

\section{Within condition results}

Since the purpose of this experiment was to compare the participants' performance among the experimental conditions, only brief details of the performance within the experimental conditions are presented here (full details of the analyses can be obtained from the authors). These details are presented to demonstrate that the participants took the tasks seriously and attempted to perform them as effectively as possible.

In the verbalization condition, there was a significant reduction (between Trials 1 and 3) in the time it took the participants to describe the route, and there was a marginally significant improvement in the accuracy of their descriptions (between Trials 1 and 3 ). In the modeling condition, the par- 
ticipants became quicker at laying out the model (between Trials 1 and 2). The models were analyzed in two ways. First, they were assessed for topological structure (e.g., start, straight section, a right turn, a straight section, zigzag, landmark, and so on), and there was a marginally significant improvement (between Trials 1 and 2). Second, bidimensional analyses were conducted to measure the goodness of fit between the models and an accurate real-world model. There was a significant improvement (between Trials 1 and 2) in the fit of the models for the severely visually impaired participants, but not for the moderately visually impaired participants.

In the pointing condition, the time to complete the pointing task declined (between Trials 1 and 3). For each participant and each pointing estimate, the difference between the pointing estimate and the true bearing (i.e., the pointing error) was calculated and converted into percentages of 180 degrees. The mean error score for each participant was calculated. There was no significant difference in the mean error scores between trials, but the lack of improvement was attributed to the fact that the pointing estimates were accurate from the first trial and that the mean error score (for all three trials) was only $18 \%$.

These results show that the participants generally became quicker and more accurate in carrying out the tasks over the course of the experiment. They were expected because the participants gained more experience of the route and more experience with the tasks during the three trials. The results are presented here to emphasize that the participants did pay attention to the tasks that they were asked to carry out in each condition.

\section{Discussion}

In this study, groups of visually impaired participants were given three trials to learn an unfamiliar route. The groups differed in the type of tests that they carried out during the trials. The participants were asked to point between places during each trial, to describe the route at the end of each trial, or to make a model of the route at the end of each trial. A control group learned the route without using any spatial tasks.

The authors predicted that the participants who carried out a spatial task would learn the route better than the participants in the control group. They did not expect any major differences between the groups during the first trial because the participants had not experienced the tasks until the end of the first trial. However, they did expect differences in performance during the later trials, after the participants had started carrying out the tasks. In summary, the authors predicted an interaction between trial and condition. This interaction, for both accuracy of route choices at all choice points and accuracy at the seven critical choice points, was only close to significant. For this reason, the results should be treated with caution and require replication. Nonetheless, an inspection of the interaction showed that the clearest effect for both measures was in the difference between the modeling group and the control group. While the control group showed little improvement between the first and second trials, the modeling group showed the largest improvement between these two trials on both measures.

Another way of describing this contrast between the modeling group and the control group is to say that the modeling group performed similarly to the control group during the first trial but were better than the control 
group during the second trial. In the third trial, the modeling group were still better than the control group, but as the control group improved most between the second and third trial, the performance of that group became closer to the performance of the modeling group. The authors therefore concluded that the modeling task had some effect on learning.

As was pointed out at the beginning of the article, many studies of people who are learning new environments have used tasks like the ones used in this experiment to measure route knowledge. Since the authors demonstrated an influence of spatial tasks on learning performance, the findings raise a general methodological issue. There is no reason to expect that completing a spatial task will confound learning if it is used only once. However, if it is carried out repeatedly, there is the danger that performing the task itself will contribute to the learning process. How crucial is repeated testing? It was found that the effect of repeated testing had the most influence between Trials 1 and 2 , but no effect by Trial 3. By Trial 3, all the groups were performing well, and it may be that when learning is nearly complete, the effects of any additional testing are minor. Thus, it can be speculated that the effects of testing apply mainly to the early phases of learning. Because the present experiment included only participants with visual impairments, the issue of task effects on the development of sighted people's route knowledge remains to be investigated, and the authors plan to conduct such studies in the future.

What are the implications for mobility instruction? This study showed that learning a route can be enhanced by carrying out a related spatial task, and that of the three tasks used, modeling was the most effective. Although the effect of carrying out the modeling task was only small, such a task can be used to help people with visual impairments learn new routes. Not only did modeling provide a slight benefit to the participants, it is also the type of task that can be carried out after traveling a route and hence does not interrupt a person's immediate experience of moving through the environment. In addition, the modeling task is easy to administer and requires only a minimum of materials - a few magnetic pieces of different lengths and shapes that a person can use to make a map of the environment. In previous experiments, the authors found that people who are visually impaired do not have any difficulty understanding the concept of using magnetic pieces to make a plan of a route (Ungar et al., 1997) and that they can often make models as accurately as can sighted people (Golledge et al., 2000; Jacobson et al., 1998).

Unlike previous studies that found little difference in the route-learning performance of totally blind and visually impaired participants after repeated trials (e.g., Jacobson et al., 1998), this study found that the moderately visually impaired participants performed better than did the severely visually impaired participants. However, the difference between the two groups of participants was small, and both groups had scores between 1 (indicating perfect performance) and 2 (indicating very good performance). In other words, like previous studies (e.g., Golledge et al., 2000; Jacobson et al., 1998), the authors found generally high levels of routelearning performance by all the groups, and this finding reinforces the argument made 
at the beginning of the article-that people with visual impairments can learn their way along complex routes after relatively little exposure.

\section{References}

Espinosa, A., Ungar, S., Ochaíta, E., Blades, M., \& Spencer, C. (1998). Comparing methods for introducing blind and visually impaired people to unfamiliar urban environments, Journal of Environmental Psychology, 18, 277-287.

Golledge, R. G. (Ed.). (1999). Wayfinding behavior: Cognitive mapping and other spatial processes. Baltimore, MD: Johns Hopkins University Press.

Golledge, R. G., Jacobson, D., Kitchin, R., \& Blades, M. (2000). Cognitive maps, spatial abilities and human wayfinding. Geographical Review of Japan, 73 (Series B), 93-104.

Jacobson, D., Kitchin, R., Garling, T., Golledge, R. G., \& Blades, M. (1998). Learning a complex urban route without sight: Comparing naturalistic versus laboratory methods: Annual Conference of Cognitive Science Society of Ireland.

Kitchin, R., \& Blades, M. (2001). The cognition of geographic space. London: Tauris.

Kitchin, R., \& Freundschuh, S. (Eds.). (2000). Cognitive mapping: Past, present, and future. London: Routledge.

Kitchin, R., \& Jacobson, R. D. (1997) Techniques to collect and analyze the cognitive map knowledge of persons with visual impairment or blindness: Issues of validity. Journal of Visual Impairment \& Blindness, 91, 360-376.
Ochaíta, E., \& Huertas, J. A. (1993). Spatial representation by persons who are blind: A study of the effects of learning and development. Journal of Visual Impairment \& Blindness, $87,37-41$.

Passini, R., \& Proulx, G. (1988). Wayfinding without vision: An experiment with congenitally totally blind people. Environment and Behavior, 20, 227-252.

Thinus-Blanc, C., \& Gaunet, F. (1997). Representation of space in blind persons: Vision as a spatial sense? Psychological Bulletin, 121, 20-42.

Ungar, S. (2000). Cognitive mapping without visual experience. In R. Kitchin \& $S$. Freundschuh (Eds.), Cognitive mapping: Past, present, and future. London: Routledge.

Ungar, S., Blades, M., \& Spencer, C. (1997). Strategies for knowledge acquisition from cartographic maps by blind and visually impaired adults. Cartographic Journal, 34, 93-110.

Mark Blades, Ph.D., senior lecturer, Department of Psychology, University of Sheffield, Sheffield, S10 2TP, England; e-mail: <M.Blades@Sheffield.ac.uk>. Yvonne Lippa, Ph.D., research assistant, Department of Geography, University of California at Santa Barbara, Santa Barbara, CA 93106; e-mail: <lippa@psych.ucsb.edu>. Reginald G. Golledge, Ph.D., professor, Department of Geography, University of California at Santa Barbara, CA 93106 email <golledge@geog.ucsb.edu>; R. Daniel Jacobson, Ph.D., assistant professor, Department of Geography, Florida State University, Tallahassee, FL 32306; e-mail: <djacobso@garnet.acns.fsu.edu>. Robert M. Kitchin, Ph.D., senior lecturer, Department of Geography, National University of Ireland, Maynooth, County Kildare, Ireland; e-mail: $<$ rob.kitchin@may.ie>. 
Copyright of Journal of Visual Impairment \& Blindness is the property of American Foundation for the Blind. The copyright in an individual article may be maintained by the author in certain cases. Content may not be copied or emailed to multiple sites or posted to a listserv without the copyright holder's express written permission. However, users may print, download, or email articles for individual use. 